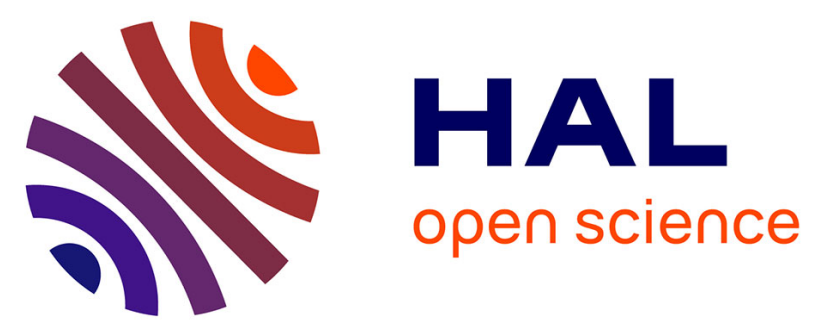

\title{
Propriétiés électrochimiques des dérivés polyconjugués hétérocycliques d'interêt biologique II. Effets des paramètres expérimentaux sur les caractéristiques des différentes vagues de réduction de la pyrimidine en solution aqueuse
}

Daniel R. Thévenot

\section{To cite this version:}

Daniel R. Thévenot. Propriétiés électrochimiques des dérivés polyconjugués hétérocycliques d'interêt biologique II. Effets des paramètres expérimentaux sur les caractéristiques des différentes vagues de réduction de la pyrimidine en solution aqueuse. Journal of electroanalytical chemistry and interfacial electrochemistry, 1973, 46, pp.89 - 107. 10.1016/S0022-0728(73)80181-0 . hal-01179288

\author{
HAL Id: hal-01179288 \\ https://hal.science/hal-01179288
}

Submitted on 23 Jul 2015

HAL is a multi-disciplinary open access archive for the deposit and dissemination of scientific research documents, whether they are published or not. The documents may come from teaching and research institutions in France or abroad, or from public or private research centers.
L'archive ouverte pluridisciplinaire HAL, est destinée au dépôt et à la diffusion de documents scientifiques de niveau recherche, publiés ou non, émanant des établissements d'enseignement et de recherche français ou étrangers, des laboratoires publics ou privés. 


\title{
PROPRIÉTÉS ÉLECTROCHIMIQUES DES DÉRIVÉS POLYCONJUGUÉS HÉTÉROCYCLIQUES D'INTERÊT BIOLOGIQUE
}

\author{
II. EFFETS DES PARAMÈTRES EXPÉRIMENTAUX SUR LES CARACTÉ- \\ RISTIQUES DES DIFFÉRENTES VAGUES DE RÉDUCTION DE LA PYRI- \\ MIDINE EN SOLUTION AQUEUSE
}

\author{
DANIEL THÉVENOT \\ Laboratoire d'Energétique Biochimique, Université Paris-Val de Marne, Avenue du Général de Gaulle, \\ 94000 Créteil (France)
}

(Reçu le 9 novembre 1972; en forme revisée le 15 mars 1973)

\section{INTRODUCTION}

La réduction électrochimique de la pyrimidine en solution aqueuse a été fréquemment étudiée car ce composé est le modèle le plus simple des hétérocycles nucléiques. Les potentiels de réduction relevés étant toujours très négatifs, seuls mercure et graphite ont pu être utilisés comme matériau d'électrode. Cavalieri et Lowy $^{1}$ puis Smith et Elving ${ }^{2-4}$ ont utilisé la polarographie sur goutte de mercure en stillation libre. Plus récemment $O^{\prime}$ Reilly et Elving ${ }^{5}$ et Dryhurst et Elving ${ }^{6}$ ont utilisé la polarographie à tension alternative surimposée et la voltamétrie sur goutte de mercure pendante et sur disque de graphite immobile.

Les auteurs ont présenté les résultats obtenus sous forme de diagramme faisant apparaître les variations avec le $\mathrm{pH}$ du potentiel de demi-vague (polarographie), de demi-pic (voltamétrie) ou de pic (polarographie à tension alternative surimposée). Nous avons rassemblé sur la Fig. 1, les résultats obtenus par ces différentes méthodes. Les points du diagramme $E_{\frac{1}{2}}-\mathrm{pH}$ obtenus par polarographie sur goutte de mercure en stillation libre sont répartis sur cinq segments de droite notés I à $\mathrm{V}(--$ ). La polarographie à tension alternative surimposée (----) et la voltamétrie sur goutte de mercure pendante (.....) ont fourni des points du diagramme potentiel-pH qui sont aussi répartis sur cinq segments de droite. L'accord entre les résultats obtenus sur mercure avec ces différentes techniques est excellent (inférieur à $50 \mathrm{mV}$ ) pour les premières étapes en milieu respectivement acide (I) et neutre (III); les divergences sont plus importantes (30 à $130 \mathrm{mV})$ pour les secondes étapes en milieu acide (II) et neutre (IV) et surtout pour les résultats en milieu basique $(\mathrm{V})(100$ à $200 \mathrm{mV})$. L'emploi de graphite immobile (-.-.) ne permet pas d'atteindre des potentiels aussi négatifs que le mercure et entraine semble-t-il une diminution du potentiel de la première étape de réduction en milieu acide $(-50$ à $-120 \mathrm{mV})$ et surtout en milieu neutre $(-140$ à $-190 \mathrm{mV})$. Enfin, les potentiels de demi-pics relevés par voltamétrie, tant sur goutte de mercure que sur disque de graphite, diminuent de 50 à $90 \mathrm{mV}$ si la vitesse de balayage des potentiels augmente d'une décade ce qui montre que le processus réactionnel n'est pas un simple transfert rapide d'électron. 


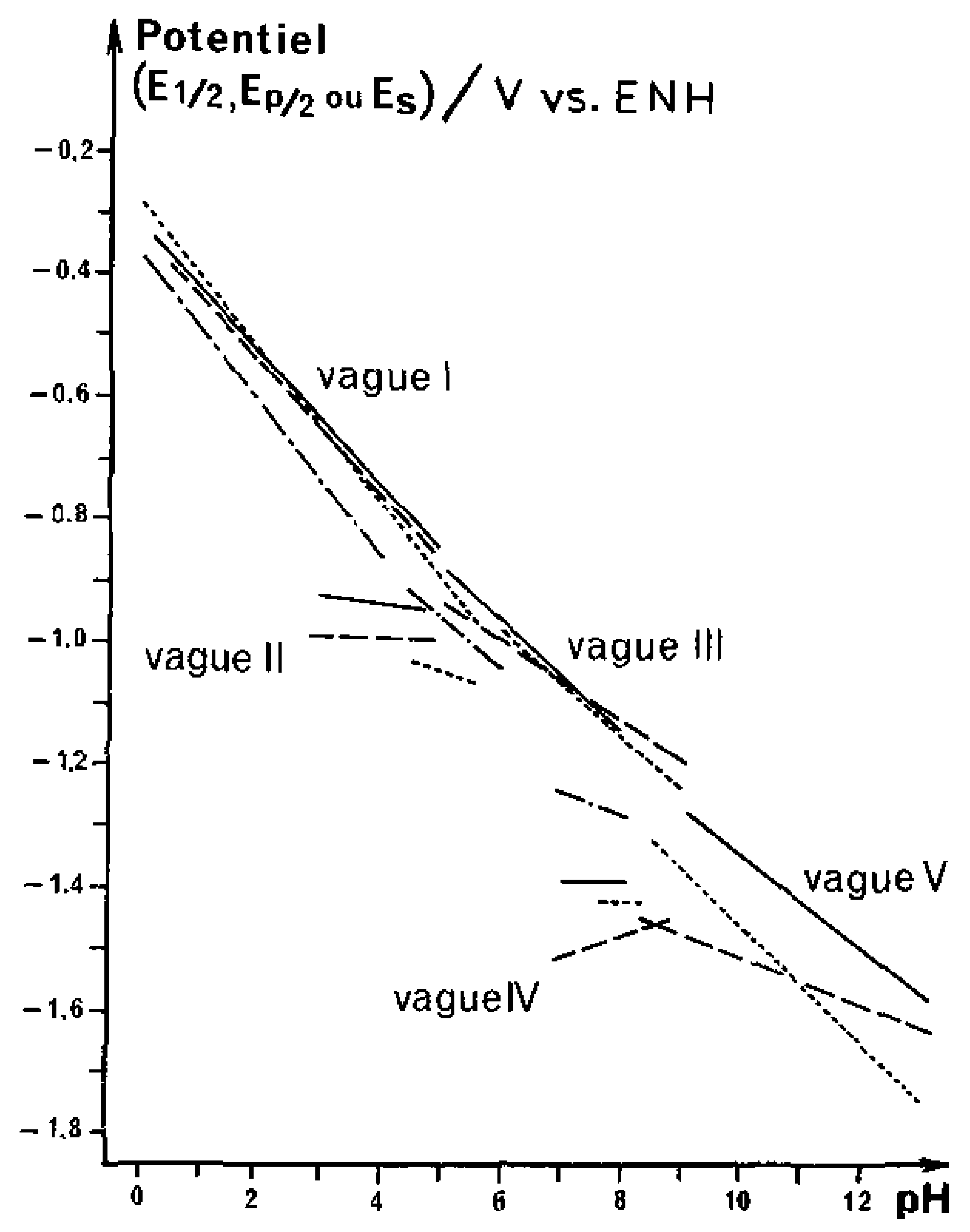

Fig. 1. Réduction électrochimique de la pyrimidine en solution aqueuse: influence du $\mathrm{pH}$ sur les potentiels relevés par polarographie classique sur goutte de mercure à stillation libre $(-)^{1-4}$, par polarographie à tension alternative surimposée $(----)^{5,6}$ et par voltamétrie sur goutte de mercure pendante $(\ldots . .$.$) à$ $0.026 \mathrm{~V} \mathrm{~s}^{-1} 6$ et sur disque de graphite immobile $(-.-\cdot)$ à $0.060 \mathrm{~V} \mathrm{~s}^{-16}$.

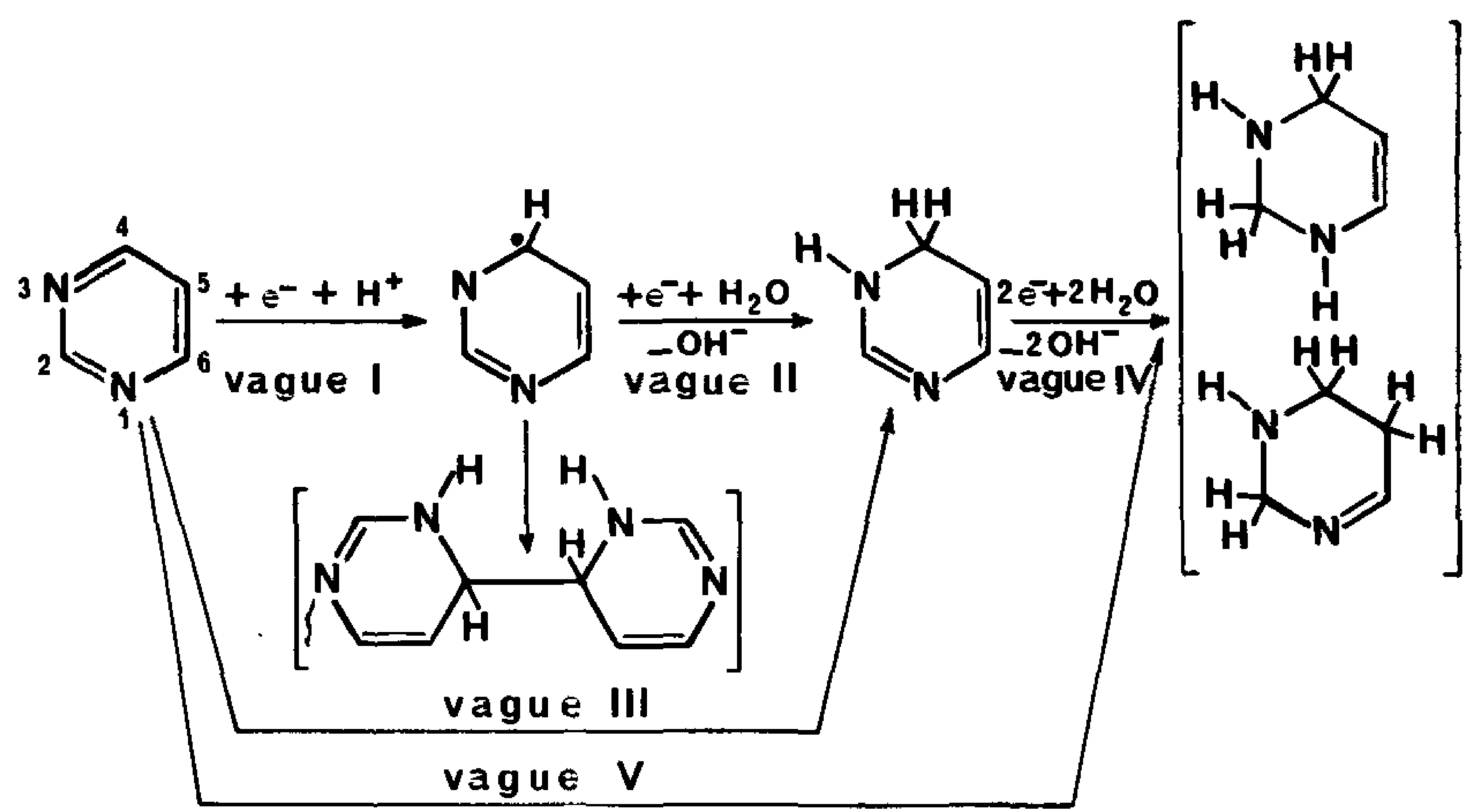

Fig. 2. Mécanismes proposés par Elving et al. ${ }^{2,4.5}$ pour interpréter les résultats de la réduction polarographique de la pyrimidine en solution aqueuse.

La comparaison des potentiels, des hauteurs et des formes des différentes vagues polarographiques de la pyrimidine en solution aqueuse, les coulométries à potentiel imposé et les relevés par spectrophotométrie ultraviolette des modifications obtenues au cours de ces coulométries, ont conduit Elving ${ }^{1,2,4}$ à proposer des mécanismes de réaction pour chacune des étapes de réaction relevées sur électrode de mercure (Fig. 2). La prise en considération de ces mécanismes pose toutefois quelques problèmes importants.

(a) Ni le radical libre, ni le dimère susceptibles d'être formés au cours du processus correspondant à la vague I n'ont jamais été décelés en solution aqueuse. 
La résonance paramagnétique électronique, effectuée dans une cellule d'électrolyse n'a fournit aucun signal caractéristique d'un radical libre ${ }^{7}$ et la voltamétrie cyclique, même à balayage très rapide, n'a donné aucun pic de réoxydation en solution aqueuse ${ }^{6}$. Une étude récente de la réduction électrochimique de la pyrimidine en solution dans l'acétonitrile non tamponnée semble indiquer la formation d'un radical anion dont l'oxydation a pu être détectée ${ }^{7.8}$ par voltamétrie cyclique sur goutte de mercure pendante à vitesse de balayage supérieure à $3 \mathrm{~V} \mathrm{~s}^{-1}$. Le produit de réduction de la pyrimidine, en solution dans l'acétonitrile non tamponnée mais contenant des quantités d'acide plus ou moins fort du même ordre de grandeur que celle de la pyrimidine, est supposé identique au produit de la première vague de réduction de la pyrimidine en solution aqueuse. De même que dans l'eau, ce produit de réduction n'a pu être réoxydé à un potentiel voisin même par voltamétrie cyclique à balayage très rapide.

(b) Si le produit de réduction de la vague I, en solution aqueuse, se dimérise réellement à une vitesse raisonnable, le temps de goutte en polarographie, la vitesse de balayage en voltamétrie et la concentration de pyrimidine avec les deux techniques devrait avoir une influence non négligeable sur les potentiels des deux étapes de réduction en milieu acide (vagues ou pics I et II). A notre connaissance aucun de ces quatre effets n'a été jusqu'à présent relevé par les auteurs qui ont proposé ces mécansimes.

(c) Les potentiels des secondes étapes de réduction en milieu acide (II) ou neutre (IV) ne dépendant pratiquement pas du pH de la solution, l'hydrolyse ou la protonation des produits formés, au cours de ces deux étapes, ne peuvent pas, thermodynamiquement, se réaliser en même temps que le transfert électronique. Si ces réactions chimiques existent, elles ne peuvent donc se dérouler qu'après le transfert d'électron et à vitesse beaucoup plus lente que celui-ci.

(d) Les potentiels des étapes de réduction de la pyrimidine en solution aqueuse dépendent du $\mathrm{pH}$, ce qui indique l'intervention de transfert de protons à vitesse assez élevée avant, pendant ou après le transfert d'électrons. Ces variations $\Delta E_{\frac{1}{2}} / \Delta \mathrm{pH}$ ou $\Delta E_{\mathrm{p} / 2} / \Delta \mathrm{pH}$ ou $\Delta E_{\mathrm{s}} / \Delta \mathrm{pH}$ ne correspondent cependant pas à celles thermodynamiquement prévisibles à la température des expériences soit:

$-0.058 p / n \quad \mathrm{~V}$ unité de $\mathrm{pH}^{-1}$

si $p$ et $n$ sont respectivement le nombre de protons et d'électrons échangés.

(e) Tous les potentiels des étapes de réduction de la pyrimidine en solution aqueuse sont inférieurs au potentiel thermodynamique de réduction du solvant. Par conséquent tous les composés formés primairement à l'électrode sont susceptibles de réagir avec l'eau avec des vitesses de réaction qui peuvent dépendre des conditions expérimentales de géométrie d'électrode, d'apport de réactif à l'électrode, etc. Il faut donc être très prudent dans l'usage des bilans globaux de réaction de macro-électrolyse à potentiel imposé, pour interpréter les processus observés en polarographie ou en voltamétrie.

En conclusion, pour accéder à une connaissance des mécanismes de réduction des hétérocycles nucléiques et en particulier de la pyrimidine, telle que les hypothèses faites puissent être considérées comme solides, de nombreuses données sont encore absentes ou insuffisantes. Nous avons donc envisagé de combler au moins partiellement cette lacune en cherchant à disposer d'une quantité aussi importante que 
possible de données concernant les processus électrochimiques qui définissent les propriétés de réduction de la pyrimidine. Ces données ont été recherchées dans l'étude détaillée de l'influence sur le potentiel, la hauteur et la forme des vagues ou pics de réduction de la pyrimidine en solution aqueuse, des paramètres expérimentaux suivants: $\mathrm{pH}$ de la solution, nature et concentration des espèces tamponnant le $\mathrm{pH}$ de la solution, concentration de la pyrimidine, conditions d'apport de réactif à l'électrode (débit de mercure et temps de goutte imposé en polarographie, vitesse de balayage des potentiels en voltamétrie), température de la solution, nature du matériau d'électrode (mercure ou carbone vitreux).

\section{PARTIE EXPÉRIMENTALE}

\section{Réactifs}

Nous avons employé pour préparer les solutions tampons des réactifs de pureté analytique. Les concentrations des espèces tamponnant le $\mathrm{pH}$ sont au moins cinquante fois plus élevées que celle du réactif étudié: le plus souvent les concentrations des formes acides et basiques sont prises toutes deux égales à $0.5 \mathrm{M}$.

La pyrimidine utilisée, de provenance Fluka, Mann, Aldrich et Schuchardt, est de pureté analytique. Son comportement polarographique n'a indiqué la présence d'aucune trace d'impureté électroactive.

\section{Appareillage}

Polarographie à stillation commandée. L'ensemble de mesures utilisé comprend essentiellement des appareils Tacussel et un enregistreur Sefram. Il est composé de:

un potentiostat PRT $500 \mathrm{LC}$ imposant un potentiel défini entre une électrode de référence et une électrode indicatrice;

un millivoltmètre électronique $S 6 \mathrm{RZ}$ à grande impédance d'entrée dont le signal de sortie est envoyé à la partie tension (X) d'un enregistreur XY;

un pilote de tension Servovit 9B permettant le balayage linéaire en fonction du temps du potentiel imposé par le potentiostat (vitesse réglable continûment

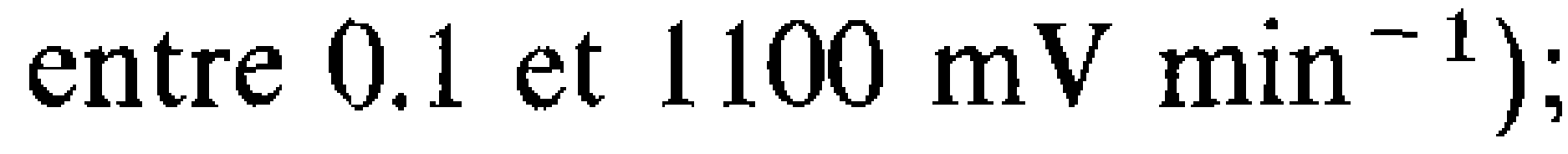

un marteau électrique MPO commandé par un générateur de commande de marteau à répétition GCMR qui fixe le temps de goutte continûment entre 0.01 et $100 \mathrm{~s}$.

L'enregistreur bigalvanométrique utilisé est un Luxytrace TR VAC Sefram branché pour sa partie courant $(\mathrm{Y})$ sur le circuit de l'électrode auxiliaire.

Cet ensemble expérimental permet le relevé direct de courbes intensitépotentiel avec une précision de mesure du courant de $1 \%$ et du potentiel de $\pm 2 \mathrm{mV}$.

Voltamétrie cyclique sur disque immobile de carbone vitreux. L'ensemble de mesures utilisé pour la voltamétrie cyclique comprend essentiellement des appareils Tacussel et un oscilloscope Tektronix. Il est composé de:

un potentiostat à réponse rapide PRT 20-2X;

un pilote de tension GSTP permettant un balayage aller et retour, linéaire en fonction du temps, du potentiel imposé par le potentiostat;

un millivoltmètre électronique $\mathrm{S} 6 \mathrm{RZ}$ qui permet de mesurer avec précision le potentiel initial et l'amplitude du balayage; 
deux amplificateurs différentiels ADTP qui permettent de relier les voies $X$ et $\mathrm{Y}$ de l'oscilloscope à la cellule, sans modifier les potentiels et courants à mesurer.

L'enregistreur XY utilisé est un oscilloscope à mémoire Tektronix R 564 B muni de deux tiroirs amplificateurs. Cet ensemble expérimental permet le relevé direct de courbes intensité-potentiel avec une précision de mesure du courant de $5 \%$ et du potentiel de $\pm 10 \mathrm{mV}$.

Cellules électrochimiques et électrodes. Nous avons utilisé des cellules de type classique à trois électrodes de volume de travail compris entre 3 et $5 \mathrm{ml}$. Une double paroi permet, grâce à une circulation d'eau, de maintenir constante la température de la cellule. L'oxygène dissous dans les solutions est éliminé au moyen d'un barbotage d'azote de qualité $\mathrm{R}$ utilisé sans purification supplémentaire. Après une quinzaine de minutes de barbotage, un courant d'azote est maintenu au-dessus de la solution pendant toute la durée des expériences.

Les électrodes indicatrices sont constituées suivant la nature de l'étude:

soit d'un capillaire à goutte de mercure, de temps de goutte libre compris entre 4 et $15 \mathrm{~s}$ et pour lequel on fixe mécaniquement le temps de goutte à une valeur inférieure, généralement $0.50 \mathrm{~s}$. Cette régulation mécanique élimine les variations et irrégularités du temps de goutte à potentiel très négatif;

soit d'un disque de carbone vitreux de $1.2 \mathrm{~mm}$ de diamètre.

Bien que des électrodes de référence $\mathrm{Ag} / \mathrm{AgCl}, \mathrm{KCl}$ saturé (Tacussel type $\mathrm{AgCl} 10)$ aient été généralement utilisées, tous les potentiels cités sont rapportés à l'électrode normale à hydrogène à $25.0 \pm 0.2^{\circ} \mathrm{C}$. Le potentiel fourni par les électrodes de référence est périodiquement contrôlé avec des électrodes témoins: ce potentiel est connu avec une incertitude inférieure à $1 \mathrm{mV}$.

INFLUENCE DU pH ET DE LA CONCENTRATION DE PYRIMIDINE SUR LES CARACTÉRISTIQUES DES VAGUES DE RÉDUCTION

Lorsque la concentration de pyrimidine est inférieure à quelques millimoles par litre de solution, sa réduction polarographique fait apparaître cinq vagues différentes entre $\mathrm{pH} 0$ et $10^{9-15}$ (Figs. 3 et 4 , Tableau 1). En milieu très acide on relève une seule vague dont le potentiel diminue de $100 \mathrm{mV}$ lorsque le $\mathrm{pH}$ augmente d'une unité et dont la hauteur est proportionnelle à la concentration de pyrimidine. $\mathrm{A} \mathrm{pH}$ voisin de 2 une vague II apparaît à la suite de cette vague I: son potentiel est indépendant du $\mathrm{pH}$ et sa hauteur, proportionnelle à la concentration de pyrimidine, est un peu supérieure à celle de la vague I. A pH voisin de 5.2, les vagues I et II fusionnent en donnant une vague III dont la hauteur, proportionnelle à la concentration de pyrimidine est voisine de la somme des hauteurs des vagues I et II. Le potentiel de la vague III diminue de $85 \mathrm{mV}$ lorsque le $\mathrm{pH}$ augmente d'une unité. A pH voisin de 6.6 apparaît à la suite de la vague III une vague IV. Sa hauteur, proportionnelle à la concentration de pyrimidine, est inférieure à celle de la vague III et son potentiel est indépendant du $\mathrm{pH}$. Enfin à $\mathrm{pH}$ voisin de 10 les vagues III et IV fusionnent en donnant la vague $\mathrm{V}$ de hauteur voisine de la somme des hauteurs des vagues III et IV.

Ainsi que l'ont prouvé les expériences précédentes ${ }^{9-15}$ lorsque la concentration de pyrimidine augmente dans une solution de $\mathrm{pH}$ compris entre 3.0 et 5.2, la forme de la vague II change progressivement et si cette concentration est supérieure 

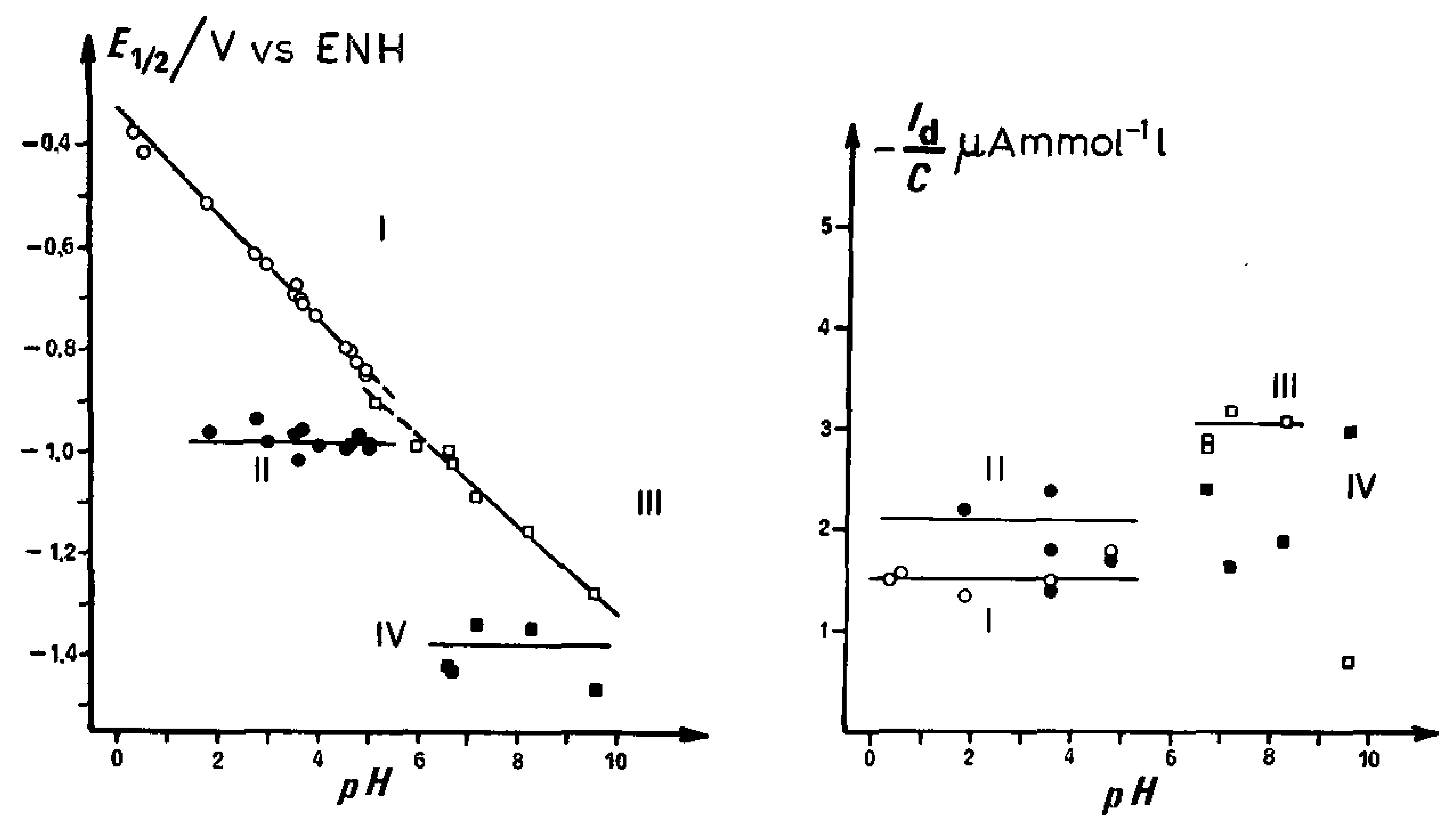

Figs. 3 et 4. Réduction de solutions aqueuses $\left(10^{-4} M\right)$ en pyrimidine sur goutte de mercure à stillation commandée. (O) Vague I, $(\bigcirc)$ vague II, ( $\square$ ) vague III et ( $\square$ ) vague IV.

Fig. 3. Influence du $\mathrm{pH}$ sur les potentiels de demi-vague.

Fig. 4. Influence du $\mathrm{pH}$ sur les hauteurs relatives des vagues. Conditions polarographiques: capillaire No. $10,35.0 \mathrm{~cm}$ de mercure, temps de goutte imposé $0.50 \mathrm{~s}$, balayage des potentiels à $250 \mathrm{mV} \mathrm{min}^{-1}$, $25.0 \pm 0.2^{\circ} \mathrm{C}$.

\section{TABLEAU 1}

INFLUENCE CONJUGUÉ DU pH ET DE LA CONCENTRATION DE PYRIMIDINE SUR SA RÉDUCTIO PAR POLAROGRAPHIE À STILLATION COMMANDÉE

\begin{tabular}{|c|c|c|c|c|c|c|c|}
\hline \multirow[t]{2}{*}{ Vague } & \multicolumn{2}{|c|}{ Domaine d'existence } & \multicolumn{3}{|c|}{ Variation de $E_{\frac{1}{2}} / m V$ vs. ENH avec le $p H$ si: } & \multirow{2}{*}{$\begin{array}{l}\frac{\Delta E_{\frac{1}{2}}}{\Delta \log c} \\
/ m V\end{array}$} & \multirow{2}{*}{$\frac{-i_{\lim }{ }^{a}}{c}$} \\
\hline & $p H$ & $\begin{array}{l}\text { Concentr. } \\
\text { de pyrimi- } \\
\text { dine } / M\end{array}$ & $c=10^{-4} M$ & $c=10^{-3} M$ & $c=10^{-2} \mathrm{M}$ & & \\
\hline I & $0.3-5.2$ & $\begin{array}{l}10^{-5}- \\
2 \times 10^{-2}\end{array}$ & $\begin{array}{l}-340-100 \mathrm{pH} \\
( \pm 15)\end{array}$ & $\begin{array}{l}-320-100 \mathrm{pH} \\
( \pm 15)\end{array}$ & $\begin{array}{l}-315-100 \mathrm{pH} \\
( \pm 20)\end{array}$ & $+20( \pm 5)$ & $\begin{array}{l}1.5_{0} \\
( \pm 0.10)\end{array}$ \\
\hline II & $2.0-5.2$ & $\begin{array}{l}10^{-5} \\
2.3 \times 10^{-3}\end{array}$ & $\begin{array}{l}-960 \\
( \pm 30)\end{array}$ & $\begin{array}{l}-1000 \\
( \pm 15)\end{array}$ & & $-35( \pm 7)$ & $\begin{array}{l}1.4_{5} \\
2.4_{0}\end{array}$ \\
\hline $\mathrm{II}_{\mathrm{a}}$ & $3.0-5.2$ & $\begin{array}{l}2.3 \times 10^{-3} \\
2 \times 10^{-2}\end{array}$ & & & $\begin{array}{l}-975 \\
(+25)\end{array}$ & $\sim 0$ & $(4.5)^{b}$ \\
\hline$I_{\mathrm{b}}$ & $3.0-5.2$ & $\begin{array}{l}2.3 \times 10^{-3} \\
2 \times 10^{-2}\end{array}$ & & & $\begin{array}{l}-1080 \\
( \pm 10)\end{array}$ & -100 & \\
\hline III & $5.2-10$ & $\begin{array}{l}10^{-5} \\
2 \times 10^{-2}\end{array}$ & $\begin{array}{l}-465-85 \mathrm{pH} \\
( \pm 15)\end{array}$ & $\begin{array}{l}-465-85 \mathrm{pH} \\
( \pm 15)\end{array}$ & $\begin{array}{l}-465-85 \mathrm{pH} \\
( \pm 30)\end{array}$ & 0 & $\begin{array}{l}3.05 \\
( \pm 0.10)\end{array}$ \\
\hline IV & $6.0-10$ & $\begin{array}{l}10^{-5} \\
2 \times 10^{-2}\end{array}$ & $\begin{array}{l}-1380 \\
( \pm 60)\end{array}$ & $\begin{array}{l}-1380 \\
( \pm 60)\end{array}$ & $\begin{array}{l}-1380 \\
( \pm 60)\end{array}$ & $( \pm 15)$ & $\begin{array}{l}1.9- \\
3.0\end{array}$ \\
\hline
\end{tabular}

${ }^{a}$ Les hauteurs des différentes vagues ont été męsurées dans les conditions expérimentales suivantes: capillaire No. $35 \mathrm{~cm}$ de mercure, temps de goutte imposé $0.50 \mathrm{~s}$, débit de mercure $0.86 \pm 0.03 \mathrm{mg} \mathrm{s}^{-1}, 25.0 \pm 0.2^{\circ} \mathrm{C}$.

${ }^{b}$ Valeur en $\mu \mathrm{A}$ de la hauteur de la vague $\mathrm{II}_{\mathrm{a}}$, dans les conditions expérimentales précédentes, lorsque la concentr tion de pyrimidine est supérieure à $2.3 \mathrm{mM}$. 
à $2.3 \times 10^{-3} M$ cette vague $\mathrm{II}$ se divise en deux vagues notées $\mathrm{II}_{\mathrm{a}}$ et $\mathrm{II}_{\mathrm{b}} \cdot \mathrm{Ce}$ dédoublement a été observé dans onze solutions tampons constituées de couples acide-base différents. Le potentiel et la hauteur de la vague $\mathrm{II}_{\mathrm{a}}$ qui ne dépendent ni du $\mathrm{pH}$ ni de la concentration de pyrimidine sont égaux à ceux présentés par la vague II à la concentration de dédoublement. La somme des hauteurs des vagues $\mathrm{II}_{\mathrm{a}}$ et $\mathrm{II}_{\mathrm{b}}$ est proportionnelle à la concentration de pyrimidine, le coefficient de proportionnalité étant égal à celui qui existe entre la hauteur de la vague II et la concentration, en solution plus diluée.

Enfin, la concentration de pyrimidine a une influence non seulement sur la hauteur mais aussi sur le potentiel des différentes vagues (Fig. 5).

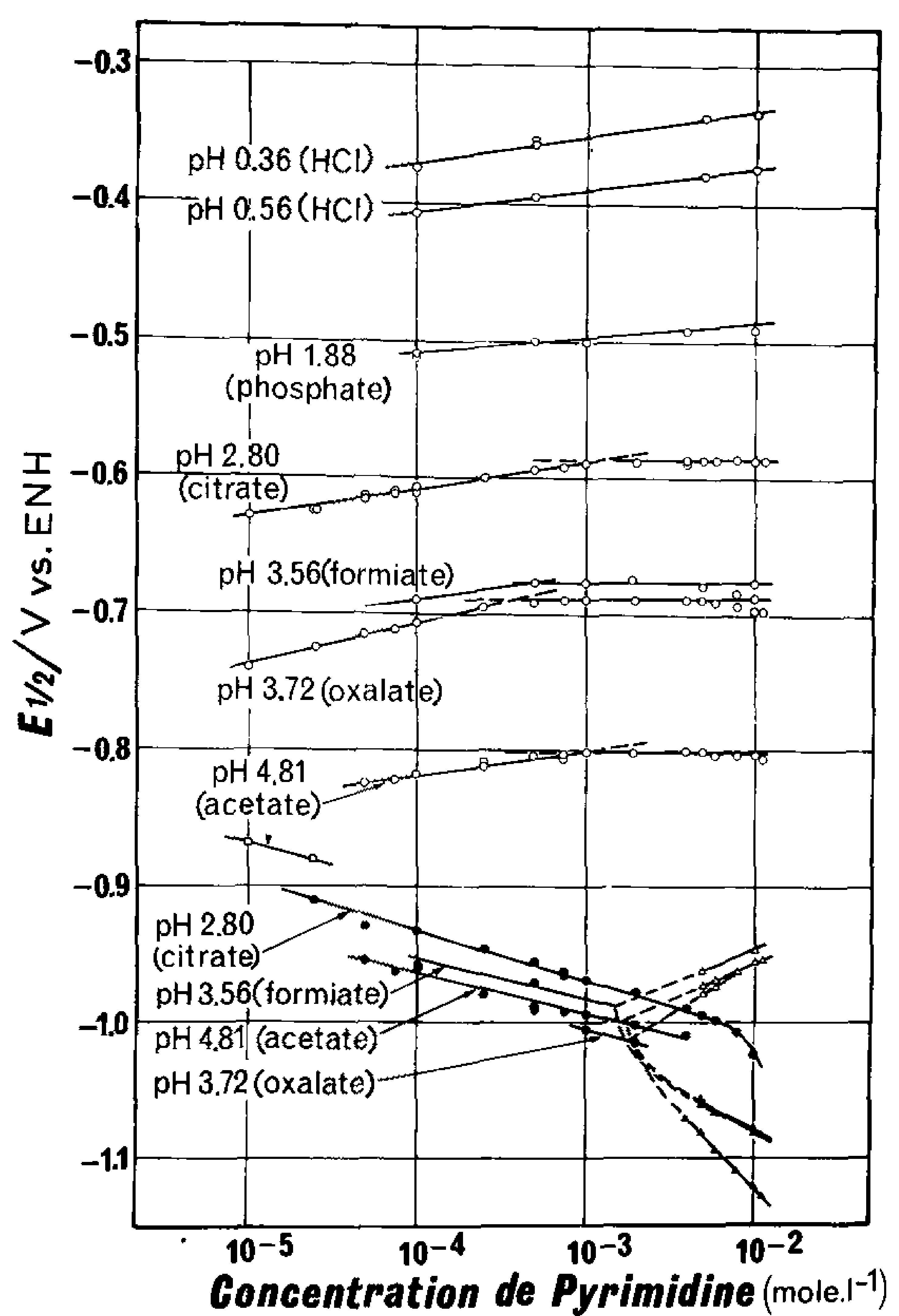

Fig. 5. Influence de la concentration de pyrimidine sur sa réduction sur goutte de mercure en stillation commandée. Comportement des potentiels de demi-vague de la vague $I(\bigcirc)$, la vague II $(\bigcirc)$, la vague $\mathrm{II}_{\mathrm{a}}(\triangle)$ et la vague $\mathrm{II}_{\mathrm{b}}(\boldsymbol{\Delta})$ dans quelques tampons.

A pH compris entre 0.3 et $5.2 E_{\frac{1}{2}}$ de la vague I varie linéairement avec le logarithme de la concentration de pyrimidine. La pente de ces variations $\Delta E_{\frac{1}{2}} / \Delta$ $\log c$ est généralement de $+20 \pm 5 \mathrm{mV}$ lorsque le $\mathrm{pH}$ est inférieur à 2.8 ou que la concentration est inférieure à environ $10^{-3} \mathrm{M}$ à $\mathrm{pH}$ compris entre 2.8 et 5 . Le potentiel de demi-vague devient indépendant de la concentration si celle-ci est supérieure à environ $10^{-3} \mathrm{M}$ à $\mathrm{pH}$ compris entre 2.8 et 5 . Ce comportement est identique à celui observé pour la première vague de réduction de l'iodure de $\mathrm{N}_{1}$ 
méthyl nicotinamide en milieu neutre ou basique ${ }^{16.17}$. Il révèle l'intervention dans le mécanisme de réaction correspondant à la vague $\mathrm{I}$ :

soit d'étape de réaction, d'ordre différent de l'unité, qui limiterait la vitesse de transfert des électrons,

soit de réaction d'oxydo-réduction réversible conduisant à des produits en nombre différent des réactifs, les composés à potentiel chimique constant (solvant, solides, constituants du tampon) n'intervenant pas dans ce décompte ${ }^{18}$.

A pH compris entre 2.0 et 5.2, $E_{\frac{1}{2}}$ de la vague II varie linéairement avec le logarithme de la concentration de pyrimidine si celle-ci varie entre $10^{-5}$ et $2.3 \times 10^{-3}$ $M$ (Fig. 5). La pente de cette variation $\Delta E_{\frac{1}{2}} / \Delta \log c$ est généralement de $-35 \pm 7 \mathrm{mV}$. Ce comportement est semblable à celui observé pour la deuxième vague de réduction de l'iodure de $\mathrm{N}_{1}$ méthyl nicotinamide en milieu basique ${ }^{16.17}$. Ce glissement du $E_{\frac{1}{2}}$ de la vague II, lorsque la concentration de pyrimidine varie, révèle l'existence, dans le mécanisme de réaction électrochimique, du même type de réaction que pour la vague $I$.

Le Tableau 1 résume les résultats relatifs à l'influence conjuguée du $\mathrm{pH}$ de la solution et de la concentration de pyrimidine sur la réduction polarographique de ce composé.

\section{EFFET DE LA NATURE ET DE LA CONCENTRATION DU TAMPON}

L'analyse des résultats que nous venons de rappeler (Figs. 3 à 5) montre que ceux-ci sont indépendants de la nature et concentration de la solution tampon autrement que par le $\mathrm{pH}$ qu'elle détermine. Nous avons en effet utilisé treize couples acide-base différents et des concentrations d'espèces tampon comprises entre $10^{-2}$ et $2 M$.

Par rapport à ce comportement général, nous avons relevé les quelques exceptions suivantes. Tout d'abord dans les tampons contenant neuf fois plus d'acide acétique que d'acétate, à $\mathrm{pH} 3.7$, la concentration du tampon influe considérablement sur les caractéristiques des vagues I et II. Lorsque la concentration d'acétate est égale à $10^{-2} M$, ces deux vagues se dédoublent toutes deux si la concentration de pyrimidine est supérieure à environ $10^{-4} \mathrm{M}$; au contraire lorsque les concentrations en acide acétique et acétate sont dix fois plus élevées les vagues I et II ont été observées sans discontinuité dans des solutions contenant $10^{-5}$ à $10^{-2} \mathrm{M}$ en pyrimidine. Enfin la vague III présente des comportements anormaux à pH 6.71 en tampon phosphate (dédoublement de la vague III à concentration de pyrimidine supérieure à $3.7 \mathrm{mM}$ ) et à $\mathrm{pH} 9.57$ en tampon borate (hauteur de la vague III proportionnelle à la puissance 1.3 de la concentration de pyrimide).

\section{EFFET DE LA TEMPÉRATURE DE LA SOLUTION}

L'effet de la température sur le potentiel de demi-vague et la hauteur des vagues $\mathrm{I}, \mathrm{II}, \mathrm{II}_{\mathrm{a}}$ et $\mathrm{II}_{\mathrm{b}}$ a été étudié pour des solutions environ $10^{-2} \mathrm{M}$ en pyrimidine à $\mathrm{pH} 3.72\left(7.8\right.$ et $\left.25^{\circ} \mathrm{C}\right)$ et à $\mathrm{pH} 4.77\left(10,25\right.$ et $\left.40^{\circ} \mathrm{C}\right)$.

Les potentiels de demi-vague correspondant à des transferts électroniques rapides varient généralement peu avec la température ${ }^{19-23}:-2$ à $+2 \mathrm{mV}^{\circ} \mathrm{C}^{-1}$. Nous avons observé que l'effet de la température sur les potentiels de demi-vague 
de réduction de la pyrimidine, qui ne dépend ni du $\mathrm{pH}$ de la solution ni des conditions de stillation du mercure, est compris entre ces deux limites puisque:

$$
\begin{aligned}
\Delta E_{\frac{1}{2}} / \Delta \theta & =-0.78 \pm 0.10 \mathrm{mV}^{\circ} \mathrm{C}^{-1} \text { pour la vague } \mathrm{I}, \\
& =+0.5 \pm 0.5 \mathrm{mV}{ }^{\circ} \mathrm{C}^{-1} \text { pour la vague } \mathrm{II}, \\
& =+0.79 \pm 0.08 \mathrm{mV}^{\circ} \mathrm{C}^{-1} \text { pour la vague } \mathrm{II}_{\mathrm{a}}, \\
& =+1.36 \pm 0.17 \mathrm{mV}{ }^{\circ} \mathrm{C}^{-1} \text { pour la vague } \mathrm{II}_{\mathrm{b}} .
\end{aligned}
$$

L'influence de la température sur la hauteur des vagues polarographiques est fréquemment utilisée pour obtenir des indications sur le processus qui limite leur hauteur. Si ce processus limitant est la diffusion de réactif vers l'électrode, l'accroissement relatif de la hauteur de vague $I_{\text {lim }}^{-1} \Delta I_{\text {lim }} / \Delta \theta$ est compris ${ }^{19,24-27}$ entre 1.1 et $2.3 \%{ }^{\circ} \mathrm{C}^{-1}$; si c'est une cinétique de réaction, l'accroissement relatif de la hauteur de vague est supérieur ${ }^{25-27}$ à $2.5 \%{ }^{\circ} \mathrm{C}^{-1}$; enfin si l'adsorption de réactif est ce facteur limitant, la hauteur de vague diminue généralement lorsque la température augmente ${ }^{25,27}$. Aux $\mathrm{pH} 3.7_{2}$ et $4.7_{7}$ nous avons observé que les hauteurs de vague de réduction de la pyrimidine augmentent généralement avec la température, cet effet ne dépendant ni du $\mathrm{pH}$ de la solution ni des conditions de stillation du mercure:

$$
\begin{aligned}
\frac{1}{I_{\lim }} \cdot \frac{\Delta I_{\lim }}{\Delta \theta}=\frac{\Delta \log \left(-I_{1 \mathrm{im}}\right)}{\Delta \theta} & =+1.7 \pm 0.2 \%{ }^{\circ} \mathrm{C}^{-1} \text { pour la vague } \mathrm{I} \\
& =+0.7 \text { à } 5.1 \%{ }^{\circ} \mathrm{C}^{-1} \text { pour la vague } \mathrm{II} \\
& =+0.8 \pm 0.5 \%{ }^{\circ} \mathrm{C}^{-1} \text { pour la vague } \mathrm{II}_{\mathrm{a}} \\
& =+0.6 \pm 0.7 \%{ }^{\circ} \mathrm{C}^{-1} \text { pour la vague } \mathrm{II}_{\mathrm{b}} .
\end{aligned}
$$

L'ensemble des résultats relatifs à l'effet de la température sur la réduction polarographique de la pyrimidine en milieu acide semble donc exclure l'intervention de réactions d'adsorption et de réaction chimique lente dans les processus définissant le potentiel et la hauteur des vagues $\mathrm{I}_{,} \mathrm{II}_{\mathrm{a}}$ et $\mathrm{II}_{\mathrm{b}}$.

\section{EFFET DE LA FRÉQUENCE DE CHUTE DES GOUTTES DE MERCURE}

Le temps de goutte joue en polarographie un rôle inverse de celui de la vitesse de balayage des potentiels en voltamétrie sur électrode immobile ${ }^{28}$. Afin de préciser la nature des étapes de réduction de la pyrimidine, nous avons, en maintenant constante la hauteur de la colonne de mercure au-dessus du capillaire, et donc le débit de mercure, étudié l'influence du temps de goutte imposé $\tau$ sur le potentiel et la hauteur de toutes les vagues précédemment décrites.

Pour un système électrochimique réversible comme $\mathrm{Tl}^{0} / \mathrm{Tl}^{+}$ou $\mathrm{Cd}^{0} / \mathrm{Cd}^{2+}$ la fréquence de chute des gouttes de mercure n'a aucun effet sur le potentiel de demi-vague. Dans le cas de la réduction de la pyrimidine nous avons observé par contre que les potentiels de toutes les vagues augmentent linéairement avec le logarithme du temps de goutte imposé $\tau$ (Fig. 6). 
En effet à $\mathrm{pH}$ compris entre 3.5 et 4.8

$$
\begin{aligned}
\Delta E_{\frac{1}{2}} / \Delta \log \tau & =+17.5 \pm 3 \mathrm{mV} \text { pour la vague } \mathrm{I} \\
& =+30 \pm 4 \mathrm{mV} \text { pour la vague } \mathrm{II} \\
& =+73 \pm 6 \mathrm{mV} \text { pour la vague } \mathrm{II}_{\mathrm{a}} \\
& =+26 \pm 10 \mathrm{mV} \text { pour la vague } \mathrm{II}_{\mathrm{b}}
\end{aligned}
$$

Les valeurs de ces variations ne dépendent de façon sensible ni du $\mathrm{pH}$ de la solution (3.5 à 4.8), ni de la température $\left(7\right.$ à $\left.40^{\circ} \mathrm{C}\right)$, ni de la concentration de pyrimidine $\left(10^{-4}\right.$ à $\left.10^{-2} \mathrm{M}\right)$. Il est important de remarquer l'identité des résultats obtenus pour les vagues I et II de la pyrimidine et pour les deux étapes de réduction de l'iodure de $\mathrm{N}_{1}$ méthyl nicotinamide en milieu neutre ou basique ${ }^{16,17}$.
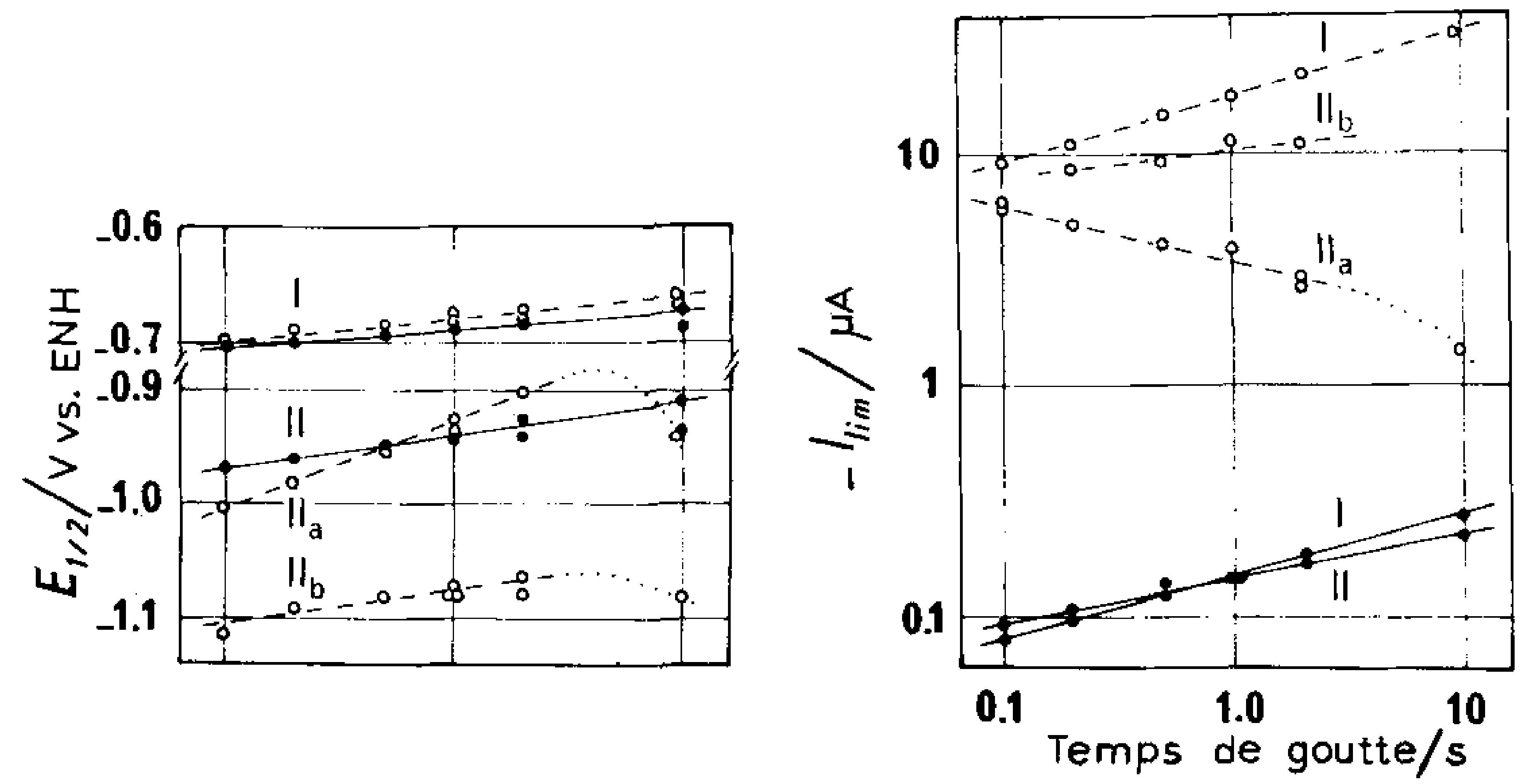

Figs. 6 et 7. Influence de la fréquence de chute des gouttes de mercure sur la réduction de la pyrimidine par polarographie à stillation commandée. Solutions $10^{-4} M(O-O)$ et $10^{-2} M\left(O^{--} \bigcirc\right)$ en pyrimidine dans un tampon formiate ( $\mathrm{pH} 3.56)$. Conditions polarographiques: capillaire No. $10.35 .0 \mathrm{~cm}$ de mercure, $25.0 \pm 0.2^{\circ} \mathrm{C}$

Fig. 6. Relations entre les potentiels de demi-vague et le logarithme du temps de goutte imposé.

Fig. 7. Relations entre le logarithme des hauteurs de vague et le logarithme du temps de goutte imposé.

La variation de la fréquence de chute des gouttes de mercure a un effet comparable sur la hauteur des vagues I et II et sur la hauteur des vagues de réduction des cations $\mathrm{Tl}^{+}$et $\mathrm{Cd}^{2+}$. En effet (Fig. 7)

$$
\begin{aligned}
\Delta \log \left(-I_{\lim }\right) / \Delta \log \tau & =+0.29 \pm 0.02 \text { pour la vague } \mathrm{I} \\
& =0.30 \pm 0.04 \text { pour la vague } \mathrm{II} \\
& =+0.25 \pm 0.07 \text { pour la réduction }
\end{aligned}
$$

de $\mathrm{Tl}^{+}$ou $\mathrm{Cd}^{2+}$, les valeurs de ces variations ne dépendant de façon sensible ni du $\mathrm{pH}$ de la solution (3.5 à 4.8), ni de la température $\left(7\right.$ à $\left.40^{\circ} \mathrm{C}\right)$, ni de la concentration de pyrimidine $\left(10^{-4}\right.$ à $\left.10^{-2} M\right)$.

Par contre, la hauteur de la vague $\mathrm{II}_{\mathrm{a}}$, qui rappelons-le est indépendante de la concentration de pyrimidine, diminue lorsque le temps de goutte augmente (Fig. 7) selon:

$$
\Delta \log \left(-I_{\lim }\right) / \Delta \log \tau=-0.14 \pm 0.03
$$


Cette pente, qui n'est pas connue avec une grande précision car la vague $\mathrm{II}_{\mathrm{a}}$ est toujours assez proche de la vague $\mathrm{II}_{\mathrm{b}}$, reste toujours négative dans tout le domaine de $\mathrm{pH}(3.5$ à 4.8$)$ et de température $\left(7\right.$ à $\left.40^{\circ} \mathrm{C}\right)$ étudié. Un tel phénomène est généralement expliqué par l'intervention d'une réaction d'adsorption limitant le transfert des électrons ${ }^{27}$.

Les résultats obtenus pour les vagues III et IV à $\mathrm{pH} 6.0$ sont qualitativement semblables à ceux relevés pour les vagues I et II en milieu acide: potentiels et logarithme des hauteurs de vague augmentent linéairement avec le logarithme du temps de goute imposé.

\section{ANALYSE LOGARITHMIQUE DE LA FORME DES VAGUES}

L'effet de la concentration de pyrimidine et du temps de goutte imposé sur la position des vagues I et II en milieu acide est identique à celui observé lors de la réduction de sels de $\mathrm{N}_{1}$ alkyl nicotinamide ${ }^{16,17}$ et de $\mathrm{N}_{1}$ alkyl pyridinium ${ }^{29,33}$, d'aldéhydes et cétones aromatiques ${ }^{30-32}$ et de sels d'immonium ${ }^{34}$. De nombreux travaux ont démontré l'intervention dans les exemples précédents d'une réaction de dimérisation radicalaire intercalée entre les deux étapes de réduction. Lorsque la vitesse de dimérisation n'est ni trop lente, ni trop rapide comparée à la vitesse de diffusion, Mairanovskii ${ }^{35}$ et Volke et $a .^{33}$ ont prouvé que la première vague polarographique a pour expression:

$$
E=E^{0}-\frac{R T}{n F} \log \frac{I^{\frac{2}{3}}}{I_{\mathrm{lim}}-I} I_{\lim }^{\frac{1}{3}}+\frac{R T}{n F} \log \frac{c k \tau}{1.51}
$$

et que par conséquent à $25^{\circ} \mathrm{C}$

$$
\Delta E_{\frac{1}{2}} / \Delta \log c=\Delta E_{\frac{1}{2}} / \Delta \log \tau=+19.7 \mathrm{mV}
$$

La valeur numérique que nous avons déterminée pour ces deux coefficients, respectivement $+20 \pm 5$ et $+17.5 \pm 3 \mathrm{mV}$ montre donc que la vague I correspondrait à un transfert électronique suivi d'une dimérisation à vitesse limitée. Mairanovskii ${ }^{35}$ a d’autre part montré que dans ce cas la deuxième vague devrait avoir pour expression:

$$
E=\text { constante }-\frac{2}{3} \frac{R T}{\alpha n F} \log \frac{I^{\frac{3}{2}}}{\left(I_{\text {lim }}-I\right) I_{\text {lim }}^{\frac{1}{2}}}
$$

En conséquence, nous avons recherché si les vagues I et II de réduction de la pyrimidine en milieu acide présentent une forme classique correspondant à l'expression

$$
E=\text { constante }-(R T / F) \log I /\left(I_{\text {lim }}-I\right)
$$

ou bien les formes théoriques précédentes.

A partir du tracé de la vague I obtenu à $\mathrm{pH} 1.86$ et 3.56 pour une solution $10^{-4} M$ en pyrimidine, nous avons porté $\log \left[I /\left(I_{\text {lim }}-I\right)\right](----)$ et $\log \left[I^{\frac{2}{3}} /\left(I_{\text {lim }}-I\right)\right]$ $(-)$ en fonction du potentiel. Les Figures 8 et 9 montrent que les points correspondant à la deuxième relation sont correctement alignés sur un segment de droite de pente $-1 /(48 \pm 3) \mathrm{mV}^{-1}$ assez voisine de la valeur théorique de cette pente $-1 / 59$ 

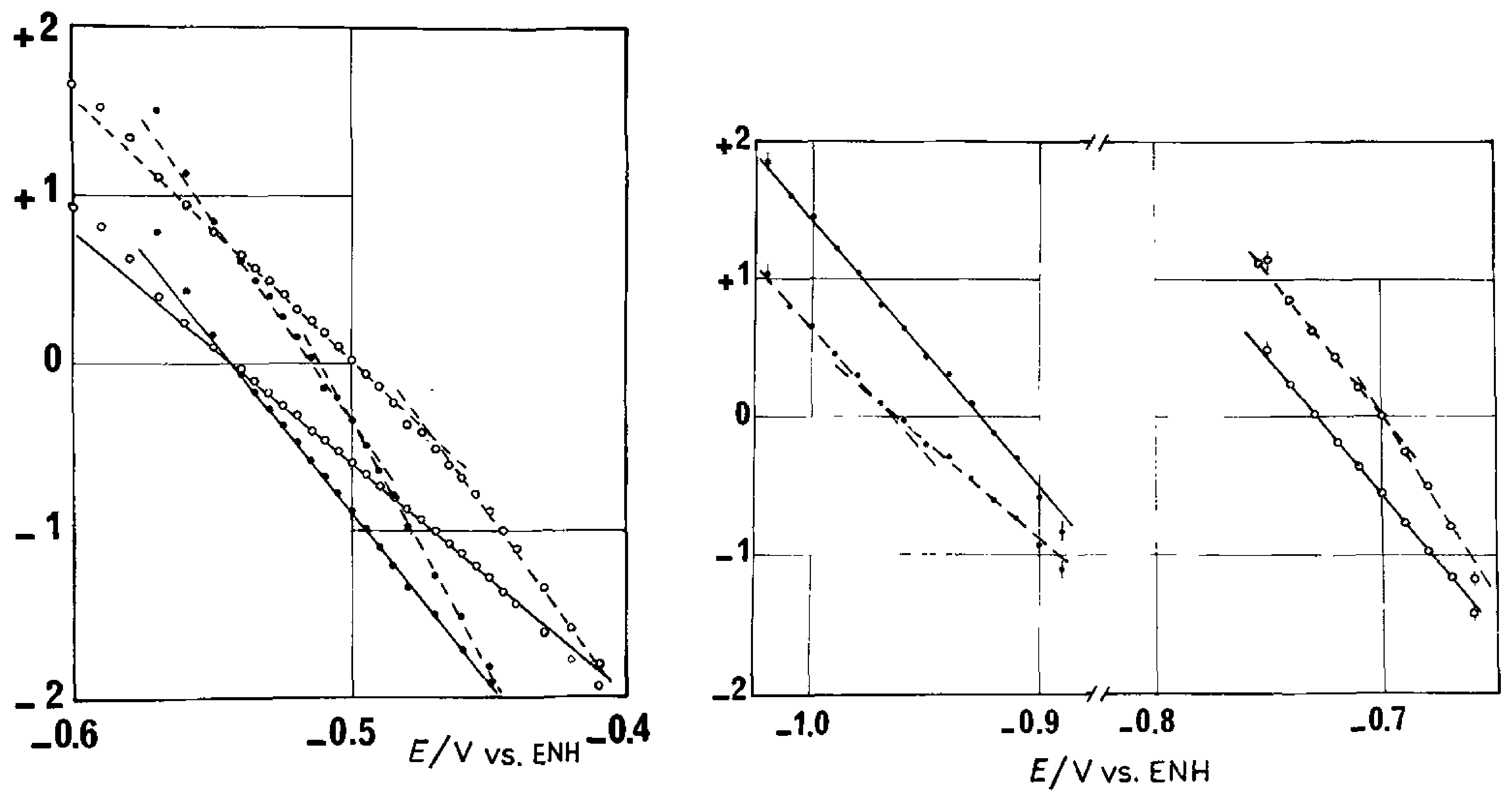

Fig. 8. Analyse logarithmique de la forme de la vague I de réduction de solutions $10^{-4} M()^{-4}$ et $10^{-2} M(\bigcirc)$ en pyrimidine à $\mathrm{pH} 1.86$ (tampon phosphate) $\log \left\{I^{\frac{2}{3}} /\left(I_{\mathrm{im}}-I\right)\right\}$ en fonction du potentiel (- $)$ et $\log \left\{I /\left(I_{1 \mathrm{~m}}-I\right)\right\}$ en fonction du potentiel (---). Conditions polarographiques: capillaire No. $10,35 \mathrm{~cm}$ de mercure, temps de goutte imposé $0.50 \mathrm{~s}, 25.0 \pm 0.2^{\circ} \mathrm{C}$.

Fig. 9. Analyse logarithmique de la forme des deux vagues I et II de réduction de solutions $10^{-4} M$ en pyrimidine à $\mathrm{pH} 3.56$ (tampon formiate) $\log \left\{I^{\frac{2}{3}} /\left(I_{1 \mathrm{~m}}-I\right)\right\}$ en fonction du potentiel pour la vague I $(\bigcirc-\bigcirc) ; \log \left\{I^{\frac{3}{2}} /\left(I_{\text {lim }}-I\right)\right\}$ en fonction du potentiel pour la vague II $(-0) ; \log \left\{I /\left(I_{\lim }-I\right)\right\}$ en fonction du potentiel pour les vagues I $\left(\mathrm{O}^{-} \mathrm{O}\right)$ et II $\left(\mathrm{O}_{-}\right)$. Conditions polarographiques: capillaire No. $10,35 \mathrm{~cm}$ de mercure, temps de goutte imposé $0.50 \mathrm{~s}, 25.0 \pm 0.2^{\circ} \mathrm{C}$.

$\mathrm{mV}^{-1}$. Au contraire les points correspondant à la première relation sont alignés sur deux segments de droite de pente différente $-1 /(33 \pm 5) \mathrm{mV}^{-1}$ et $-1 /(44 \pm 4) \mathrm{mV}^{-1}$ situés de part et d'autre du potentiel de demi-vague. Sur la Figure 8 nous avons effectué les mêmes analyses logarithmiques à partir du tracé de la vague I obtenu à pH 1.86 pour une solution $10^{-2} M$ en pyrimidine. De même que pour les solutions $10^{-4} M$ en pyrimidine, $\log \left[I /\left(I_{\mathrm{lim}}-I\right)\right]$ ne varie pas proportionnellement au potentiel puisque la pente de ces variations est de $-1 / 43$ et $-1 / 65 \mathrm{mV}^{-1}$ de part et d'autre du potentiel de demi-vague. Egalement, de même que pour les solutions $10^{-4} \mathrm{M}$ en pyrimidine, $\log \left[I^{\frac{2}{3}} /\left(I_{\text {lim }}-I\right)\right]$ varie proportionnellement au potentiel: ces variations sont cependant plus faibles puisque leur pente vaut alors $-1 / 73 \mathrm{mV}^{-1}$.

Sur la Figure 9 nous avons porté, à partir du tracé de la vague II obtenu à pH 3.56 pour une solution $10^{-4} M$ en pyrimidine, $\log \left[I /\left(I_{\text {lim }}-I\right)\right](---)$ et $\log$ $\left[I^{\frac{3}{2}} /\left(I_{\lim }-I\right)\right](--)$ en fonction du potentiel. Les points correspondant à la deuxième relation sont correctement alignés sur un segment de droite de pente $-1 /(47 \pm 3)$ $\mathrm{mV}^{-1}$. La valeur obtenue diffère de celle qui a été théoriquement établie par Mairanovskii dans le cas d'un transfert lent d'électron, concurrent avec une dimérisation intervenant au voisinage de l'électrode, soit $-1 / 80 \mathrm{mV}^{-1}$. Les points correspondant à la première relation sont alignés sur deux segments de droite de pentes différentes $-1 /(73 \pm 3) \mathrm{mV}^{-1}$ et $-1 /(55 \pm 3) \mathrm{mV}^{-1}$ situés de part et d'autre du potentiel de demi-vague.

Ces résultats montrent que les vagues I et II ne correspondent pas à un 
simple transfert électronique rapide ou lent, mais que fort probablement une réaction de dimérisation s'intercale entre ces deux étapes de réduction.

\section{COURBES ÉLECTROCAPILLAIRES ET COURANTS DE CHARGE}

L'effet de la fréquence de chute des gouttes de mercure sur la hauteur de la vague $\mathrm{II}_{\mathrm{a}}$ ainsi que son indépendance de la concentration de pyrimidine nous ont conduit à supposer l'existence d'une réaction d'adsorption limitant la vitesse de transfert des électrons. Nous avons par conséquent étudié les variations de la durée de vie des gouttes de mercure en chute libre en fonction de leur potentiel dans des solutions de pyrimidine de concentration croissante. Dans un tampon formiate de pH 3.5 nous n'avons relevé aucun changement significatif de ces courbes électrocapillaires même si la concentration de pyrimidine atteint $10^{-2} M$, valeur pour laquelle le dédoublement de la vague $\mathrm{II}$ en $\mathrm{II}_{\mathrm{a}}$ et $\mathrm{II}_{\mathrm{b}}$ est pourtant évident.

Mairanovskii ${ }^{36}$ a d'autre part montré que l'existence d'adsorption sur l'électrode entraîne fréquemment la diminution du courant de charge surtout lorsque le temps de goutte imposé est assez court et que par conséquent la fraction capacitive du courant de fond est élevée. Dans le cas de la pyrimidine, nous n'avons jamais relevé de changement significatif de ce courant de fond même dans des solutions relativement concentrées en ce réactif.

\section{EFFET DE LA NATURE DU MATÉRIAU D'ÉLECTRODE}

Afin de préciser dans quelle mesure le matériau d'électrode intervient dans les processus de réduction de la pyrimidine en solution aqueuse, nous avons comparé les résultats de mesures, stationnaires ou non, effectuées sur mercure ou carbone vitreux.

Les courbes intensité-potentiel relevées par polarographie à stillation commandée et par voltamétrie sur disque tournant de carbone sont très semblables, le décalage entre les potentiels de demi-vague étant au maximum de $50 \mathrm{mV}$ (Fig. 10).

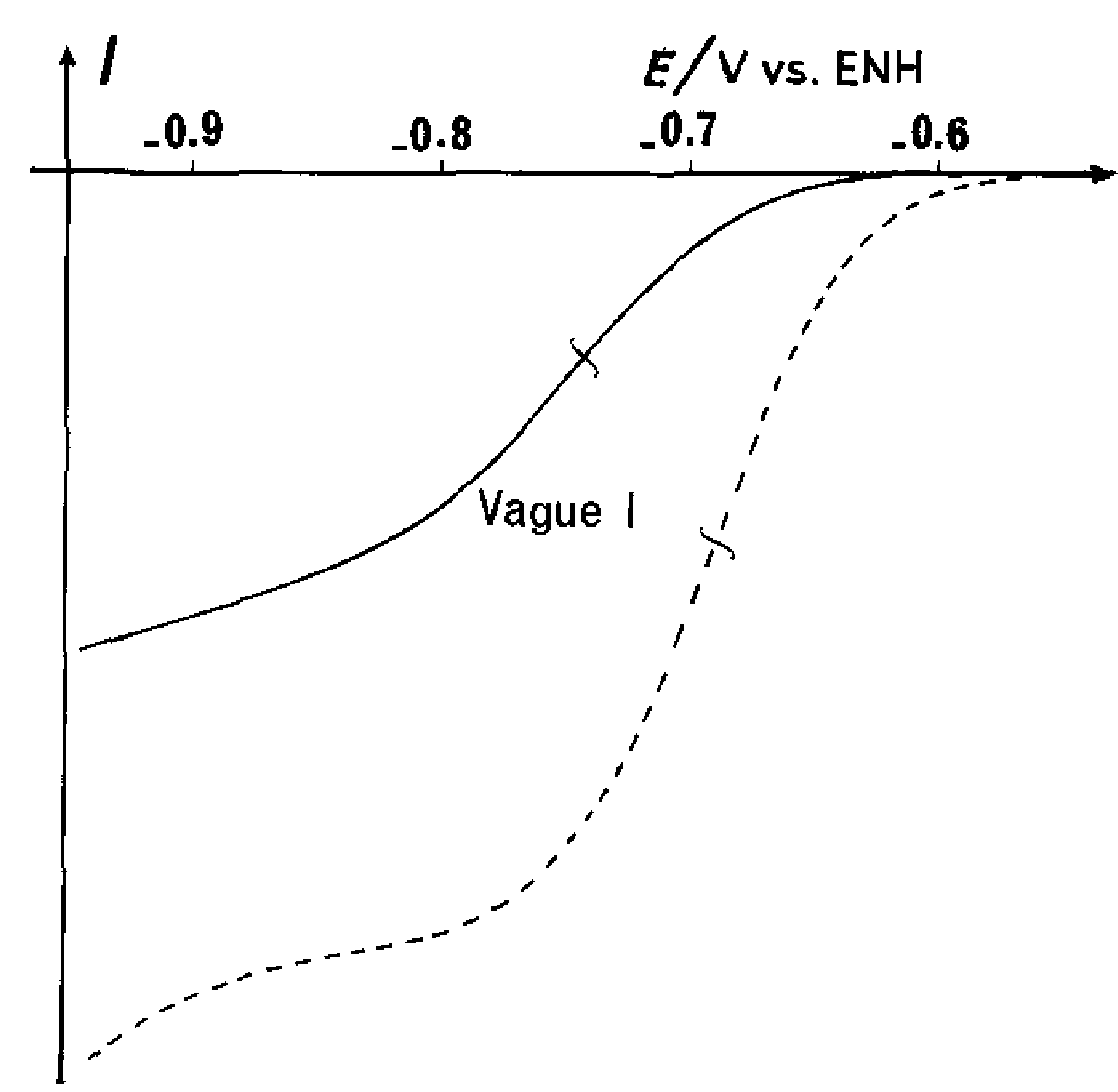

Fig. 10. Effet de la nature du matériau d'électrode sur la réduction de solutions aqueuses $10^{-2} M$ en pyrimidine à pH 3.56 (tampon formiate). Polarographie à stillation commandée (----) et voltamétrie sur disque de carbone vitreux tournant (- $)$. 
Les courbes intensité-potentiel relevées par voltamétrie à balayage linéaire rapide de potentiel $\left(1 \mathrm{~V} \mathrm{~s}^{-1}\right)$ sont également très voisines si l'électrode est soit une goutte de mercure soit un disque immobile de carbone vitreux: les potentiels de pics différent alors de moins de $20 \mathrm{mV}$, ce qui correspond à notre précision de mesure.

Les potentiels de pics voltamétriques sont généralement plus négatifs que les potentiels de demi-vague: l'écart observé $(130 \pm 30 \mathrm{mV})$ est supérieur aux 28.5 $\mathrm{mV}$ théoriques pour une fixation réversible d'un électron. Ceci correspond sans doute à une irréversibilité des réductions ou à l'intervention d'une réaction chimique comme par exemple une dimérisation.

\section{DISCUSSION ET INTERPRÉTATION DES RÉSULTATS}

\section{Vague I}

A pH inférieur à 6 la première étape de réduction de la pyrimidine, tant sur goutte de mercure à stillation commandée que sur disque de carbone vitreux, est celle pour laquelle les résultats sont les plus nombreux et les plus concordants.

L'existence d'un effet du $\mathrm{pH}$ sur le potentiel de cette vague montre que la réduction fait intervenir un échange de protons avant ou peu après le transfert des électrons. La valeur de cet effet $\left(\Delta E_{\frac{1}{2}} / \Delta \mathrm{pH}=100 \mathrm{mV}\right)$ ne permet cependant pas de conclure quant au nombre de protons fixés. L'absence de cassure des diagrammes potentiel-pH et courant- $\mathrm{pH}$ au voisinage $\mathrm{du} \mathrm{pK}_{\mathrm{a}}$ de la pyrimidine $^{1,3}$ semble exclure l'intervention d'une protonation de la pyrimidine préalable à sa réduction. L'analyse logarithmique de la forme de cette vague I, en accord avec les coulométries effectuées sur son palier de diffusion ${ }^{1.4}$ permet de supposer qu'un seul électron est alors fixé sur une molécule de pyrimidine. On aurait donc:

$$
\mathrm{P}+e^{-}+1 \text { ou } 2 \mathrm{H}^{+} \rightarrow \mathrm{PH}^{\cdot} \text { ou } \mathrm{PH}_{2}^{+}
$$

La valeur de l'effet de la concentration de réactif, du temps de goutte et de la température sur la hauteur de la vague I prouve que le processus limitant cette réaction, comme pour la réduction de $\mathrm{Tl}^{+}$ou $\mathrm{Cd}^{2+}$, est la diffusion du réactif vers l'électrode.

L'analyse logarithmique de la forme de la vague I et l'existence d'un effet de la concentration $c$ de réactif et du temps de goutte imposé $\tau$ sur le potentiel de demi-vague montrent d'autre part que la réduction est accompagnée par une réaction chimique. Les valeurs relevées tant pour $\Delta E_{\frac{1}{2}} / \Delta \log c$ que pour $\Delta E_{\frac{1}{2}} / \Delta \log \tau$, respectivement $+20 \pm 5$ et $+17.5 \pm 3 \mathrm{mV}$, sont en excellent accord avec la valeur théorique de ces deux coefficients $(+19.7 \mathrm{mV})$ qui correspond à une réaction consécutive de dimérisation. Le dimère ayant probablement pour structure $\mathrm{PH}-\mathrm{PH}$ plusieurs mécanismes peuvent être envisagés pour passer de la pyrimidine $\mathrm{P}$ à ce dimère $\mathrm{PH}-\mathrm{PH}$

soit: $\mathrm{P}+e^{-} \rightarrow \mathrm{P}^{-}, \mathrm{P}^{-}+\mathrm{H}^{+} \rightarrow \mathrm{PH}^{*}, 2 \mathrm{PH}^{\cdot} \rightarrow \mathrm{PH}-\mathrm{PH}$

soit: $\mathrm{P}+e^{-} \rightarrow \mathrm{P}^{-}, \mathrm{P}^{-}+\mathrm{H}^{+} \rightarrow \mathrm{PH}^{*}, \mathrm{P}^{-}+\mathrm{PH}^{\cdot} \rightarrow \mathrm{PHP}^{-}, \mathrm{PHP}^{-}+\mathrm{H}^{+} \rightarrow \mathrm{PH}-\mathrm{PH}$

soit: $\mathrm{P}+e^{-} \rightarrow \mathrm{P}^{-}, \mathrm{P}^{-}+2 \mathrm{H}^{+} \rightarrow \mathrm{PH}_{2}^{+}, \mathrm{P}^{-}+\mathrm{PH}^{+} \rightarrow \mathrm{PH}-\mathrm{PH}$ 
Les données disponibles ne permettent pas de choisir entre ces différentes successions de réactions celle qui s'effectue réellement. Notons que la vitesse de dimérisation n'est pas assez élevée pour être énergétiquement intégrée à la réduction. En effet si cela était le cas, $E_{\frac{1}{2}}$ augmenterait de $60 \mathrm{mV}$ par décade de concentration et non de $20 \mathrm{mV}$.

\section{Vague II}

Aux $\mathrm{pH}$ compris entre 2 et 6 , la deuxième étape de réduction de la pyrimidine en solution aqueuse est située à un potentiel si négatif qu'elle ne peut être observée que sur électrode de mercure. Ses caractéristiques sont tellement complexes qu'il n'est pas possible de définir complètement et avec sûreté les mécanismes de réaction qui lui correspondent. La similitude des hauteurs des vagues I et II conduit à penser que, lors de cette deuxième étape de réduction, un seul électron est à nouveau fixé sur une molécule de pyrimidine. L'absence d'effet du $\mathrm{pH}$ sur le potentiel et la hauteur de la vague II montre que la réduction ne fait intervenir d'échange de proton ni avant, ni après le transfert des électrons. Les valeurs de l'effet de la concentration de réactif, du temps de goutte et de la température sur la hauteur de la vague II prouvent que le processus limitant la réaction, comme pour la réduction de $\mathrm{Tl}^{+}$et $\mathrm{Cd}^{2+}$, est la diffusion du réactif vers l'électrode.

L'analyse logarithmique de la forme de la vague II et l'existence d'effets de la concentration $c$ de réactif et du temps de goutte imposé $\tau$ sur le potentiel de demi-vague montrent, d'autre part, que la réduction est accompagnée par une réaction chimique. La valeur relevée pour $\Delta E_{\frac{1}{2}} / \Delta \log c$, soit $35 \pm 7 \mathrm{mV}$, est en bon accord avec la valeur théorique de ce coefficient $(40 \mathrm{mV})$ correspondant à une réaction concurrente de dimérisation. La valeur de la pente $\Delta E / \Delta \log \left\{I^{\frac{3}{2}} /\left(I_{\lim }-I\right)\right\}$ n'est cependant pas égale au double de $\Delta E_{\frac{1}{2}} / \Delta \log c$ comme il est théoriquement prévu dans un tel $\mathrm{cas}^{35}$.

Il est fort probable que le produit formé finalement après ces deux étapes de réduction soit un dérivé dihydro $\mathrm{PH}_{2}$. Une hypothèse possible pour le mécanisme correspondant à la vague II serait alors

$$
\mathrm{PH}_{2}^{+}+e^{-} \rightarrow \mathrm{PH}_{2}
$$

en concurrence avec

$$
\mathrm{PH}_{2}^{+}+\mathrm{P}^{-} \rightarrow \mathrm{PH}-\mathrm{PH}
$$

Aucun argument expérimental ne permet cependant de confirmer ou d'infirmer cette hypothèse.

\section{Vagues $I I_{\mathrm{a}}$ et $I I_{\mathrm{b}}$}

Lorsque le $\mathrm{pH}$ de la solution est compris entre 3 et 6, la vague polarographique II se dédouble dès que la concentration de pyrimidine est supérieure à $2.3 \mathrm{mM}$. La hauteur de la première vague dédoublée $\mathrm{II}_{\mathrm{a}}$ est indépendante de la concentration de pyrimidine alors que la somme des hauteurs des deux vagues est proportionnelle à cette concentration.

Un tel ensemble de phénomènes a été rencontré par Brdička et Knobloch ${ }^{37-39}$ lors de la réduction de la riboflavine et du bleu de méthylène. Il est généralement interprété comme résultant d'une adsorption du produit de réduction sur l'élec- 
trode ${ }^{27}$. Les vagues $\mathrm{II}$ et $\mathrm{II}_{\mathrm{a}}$ correspondraient donc au même processus de formation de pyrimidine réduite adsorbée sur la goutte de mercure. La hauteur constante de la vague $\mathrm{II}_{\mathrm{a}}$ serait alors déterminée par le nombre maximal de molécules de pyrimidine réduite qui pourraient être adsorbées sur l'électrode. La valeur de cette hauteur nous permettrait de connaître la surface moyenne A occupée par chaque molécule puisque

$$
I_{\mathrm{a}}=13.5 \mathrm{~nm}^{\frac{2}{3}} / A \tau^{\frac{1}{3}}
$$

si $I_{\mathrm{a}}$ est la moyenne du courant limite de la prévague $\mathrm{II}_{\mathrm{a}}$ exprimé en $\mu \mathrm{A}, n$ est le nombre d'électrons échangé, ici probablement1, $m$ est le débit de mercure exprimé en $\mathrm{mg} \mathrm{s}^{-1}, A$ est la surface occupée par chaque molécule en $\AA^{2}, \tau$ est le temps de goutte exprimé en s. En utilisant les valeurs du courant limite obtenues pour des couples de valeurs déterminées du débit de mercure et du temps de goutte imposé, nous aurions: $3.0 \leqslant A \leqslant 6.6 \AA^{2}$. Cette hypothèse est soutenue par plusieurs observations expérimentales:

La hauteur de la vague $\mathrm{II}_{a}$ dépend du débit de mercure $m$ de la même façon que la vague "normale" I et que les vagues de réduction des cations $\mathrm{Tl}^{+}$et $\mathrm{Cd}^{2+}$. Cette variation $\Delta \log \left(-I_{\lim }\right) / \Delta \log m$ a cependant pour valeur $+0.75 \pm 0.04,0.80 \pm$ 0.10 et +0.85 respectivement pour les vagues $\mathrm{II}_{\mathrm{a}}$, I de la pyrimidine et pour les vagues de $\mathrm{Tl}^{+}$et $\mathrm{Cd}^{2+}$, au lieu de la valeur théorique commune 2/3.

Contrairement aux hauteurs de toutes les autres vagues de réduction de la pyrimidine, la hauteur de la vague $\mathrm{II}_{\mathrm{a}_{\text {a }}}$ diminue lorsque le temps de goutte augmente. Le sens de cette variation est bien celui prévu par la théorie mais la valeur de $\Delta \log \left(-I_{\text {lim }}\right) / \Delta \log \tau$ obtenue pour cette vague $\mathrm{II}_{\mathrm{a}},-0.14 \pm 0.03$, est assez différente de la valeur théorique $-1 / 3$. mentales:

Cette hypothèse est par contre infirmée par trois types d'observations expéri-

La hauteur de la vague $\mathrm{II}_{\mathrm{a}}$ augmente avec la température or l'adsorbabilité des matériaux diminue généralement lorsque la température augmente, ce qui entraîne la diminution et même la disparition des vagues d'absorption.

Le courant instantané devrait diminuer au cours de la durée de vie de la goutte selon

$$
I_{\text {inst }}=2 / 30.85 m^{\frac{2}{3}} n F / A t^{\frac{1}{3}}
$$

puisque la vitesse de formation de surface nouvelle de mercure diminue selon

$$
\mathrm{d} s / \mathrm{d} t=\frac{2}{3} 0.85 m^{\frac{2}{3}} t^{-\frac{1}{3}}
$$

Ceci n'est pas le cas puisque lorsque le potentiel imposé est égal ou voisin de $E_{\frac{1}{2}}$ de la vague $\mathrm{II}_{\mathrm{a}}$, le courant instantané croît régulièrement $\Delta \log \left(-I_{\text {inst }}\right) / \Delta \log t$ ayant des valeurs semblables pour les vagues $\mathrm{I}_{\text {, }} \mathrm{II}_{\mathrm{a}}$ et $\mathrm{II}_{\mathrm{b}}$ respectivement $+0.30 \pm 0.05$, $+0.25 \pm 0.05$ et $+0.24 \pm 0.05$.

Les courbes électrocapillaires et les courants de charge ne dépendent aucunement de la présence de pyrimidine, même à concentration égale à $10^{-2} \mathrm{M}$.

L'ensemble de résultats obtenus pour les vagues $I I, I_{a}$ et $I_{b}$ laisse donc la discussion ouverte sur l'interprétation de ce phénomène de dédoublement de la deuxième vague de réduction de la pyrimidine en milieu acide. 


\section{Vague III}

Aux $\mathrm{pH}$ compris entre 6 et 10, l'existence d'un effet de ce $\mathrm{pH}$ sur le potentiel de la première vague de réduction de la pyrimidine montre que celle-ci fait intervenir un échange de protons avant ou peu après transfert des électrons. La valeur de cet effet $\left(\Delta E_{\frac{1}{2}} / \Delta \mathrm{pH}=85 \mathrm{mV}\right)$ ne permet cependant pas de conclure quant au nombre de protons fixés. La comparaison des hauteurs des vagues III et I, à même concentration, $\left\{\left(I_{\text {lim }}\right)_{\text {III }} /\left(I_{\text {lim }}\right)_{1}=2.0\right\}$ montre que l'on peu raisonnablement supposer que deux électrons sont alors fixés sur une molécule de pyrimidine. L'analyse logarithmique et la pente de cette vague ne conduisant pas à ce résultat, nous pouvons supposer que le transfert des électrons est lent et irréversible. On aurait donc:

$$
\mathrm{P}+2 e^{-}+2 \text { ou } 3 \mathrm{H}^{+} \rightarrow \mathrm{PH}_{2} \text { ou } \mathrm{PH}_{3}^{+}
$$

bien que la fixation quasi simultanée d'un tel nombre de particules paraisse improbable.

Les effets de la concentration de réactif et du temps de goutte sur la hauteur de la vague III prouvent que le processus limitant la réduction est, comme pour la réduction des cations $\mathrm{Tl}^{+}$et $\mathrm{Cd}^{2+}$, la diffusion de réactif vers l'électrode. L'absence d'effets significatifs de la concentration de réactif et du temps de goutte sur le potentiel de cette vague III montre que la réduction n'est pas comme pour la vague I accompagnée par une réaction chimique de dimérisation.

\section{Vague IV}

Aux $\mathrm{pH}$ compris entre 6 et 10, la deuxième vague de réduction de la pyrimidine présente des caractéristiques qui varient assez considérablement avec les conditions expérimentales et techniques utilisées, sans souvent qu'un comportement général puisse être dégagé. Le potentiel de cette vague IV ne semble pas dépendre du $\mathrm{pH}$ de la solution et nous pouvons donc supposer qu'elle correspond à un transfert d'électrons seuls. Le rapport de la hauteur des vagues IV et III ne conduit pas à un nombre défini d'électrons échangés puisque à une même concentration $\left(I_{\mathrm{lim}}\right)_{\mathrm{IV}} /$ $\left(I_{\text {lim }}\right)_{\text {III }}$ varie de 0.6 à 1.0 d'un $\mathrm{pH}$ à l'autre. La valeur de l'effet de la concentration et du temps de goutte sur la hauteur de cette vague IV permet seulement d'affirmer que la vitesse de réduction est limitée par la diffusion de réactif vers l'électrode.

L'ensemble, pourtant considérable, des données expérimentales relatives aux vagues de réduction de la pyrimidine en solution aqueuse, ne permet finalement pas de proposer avec.sûreté un mécanisme pour chaque étape de réaction observée. Il a cependant permis de démontrer l'existence, jusqu'alors seulement supposée, de réactions de dimérisation entre les deux étapes de réduction en milieu acide. L'utilisation de méthodes comme la voltamétrie cyclique à balayage linéaire rapide de potentiel et la voltamétrie sur électrode disque-anneau devrait permettre de complèter et de préciser les mécanismes que nous avons proposés.

\section{RÉSUMÉ}

Les caractéristiques des différentes vagues de réduction polarographique de la pyrimidine en solution aqueuse dépendent considérablement des paramètres expérimentaux suivants: $\mathrm{pH}$ de la solution, concentration de pyrimidine, température, temps de goutte imposé. Cet ensemble d'observations et les résultats d'analyses 
logarithmiques de la forme des vagues et de courbes électrocapillaires conduisent d'une part à supposer l'existence d'une dimérisation radicalaire intercalée entre les deux premières étapes de réduction en milieu acide et d'autre part à discuter l'existence de réactions d'adsorption sur la surface de l'électrode.

\section{SUMMARY}

The characteristics of the different reduction waves of aqueous solutions of pyrimidine depend considerably on the following experimental parameters: $\mathrm{pH}$, pyrimidine concentration, temperature, controlled drop-time. In relation to these observations, to logarithmic analysis of the shape of the waves and to drop-time curves, evidence is given of a dimerization step occurring between the two reduction steps in acid media; the existence of adsorption reactions is discussed.

\section{BIBLIOGRAPHIE}

1 L. F. Cavalièri et B. A. Lowy, Arch. Biochem. Biophys., 35 (1952) 83.

2 P. J. Elving, W. A. Struck et D. L. Smith, Mises Point Chim. Anal. Org., Pharm. Bromatol., 14 (1965) 141.

3 D. L. Smith et P. J. Elving, Anal. Chem., 34 (1962) 930.

4 D. L. Smith et P. J. Elving. J. Amer. Chem. Soc., 84 (1962) 2741.

5 J. E. O'Reilly et P. J. Elving, J. Electroanal. Chem., 21 (1969) 169.

6 G. Dryhurst et P. J. Elving, Talanta, 16 (1969) 855.

7 J. E. O'Reilly et P. J. Elving, J. Amer. Chem. Soc., 93 (1971) 1871.

8 P. J. Elving et S. J. Pace, Exper. Suppl., 18 (1971) 35.

9 D. Thévenot, G. Hammouya et R. Buvet, C.R., 268 (1969) 1488.

10 D. Thévenot, G. Hammouya et R. Buvet, J. Chim. Phys., 66 (1969) 1903.

11 D. Thévenot, Communication aux Journées d'Electrochimie Organique, Thiais, 1969.

12 D. Thévenot, Communication au 3ème Congrès Int. de Biophysique, Boston, Mass., 1969.

13 D. Thévenot et G. Hammouya, Exper. Suppl., 18 (1971) 647.

14 D. Thévenot, Thèse. Paris, 1971, No. C.N.R.S. AO 6164.

15 Thévenot, Diss. Abstr. Int., 33(3) (1971).

16 D. Thévenot et G. Hammouya, Exper. Suppl., 18 (1971) 631.

17 D. Thévenot et R. Buvet, J. Electroanal. Chem., 39 (1972) 447.

18 L. Meites. Polarographic Techniques, Interscience, New York, 2e edn.. 1965, pp. 288-289.

19 G. Charlot et R. Gauguin, Les Méthodes d'Analyse des Réactions en Solution, Masson, Paris, 1951. p. 124.

20 G. Charlot, J. Badoz-Lambling et B. Tremillon, Les Réactions Electrochimiques, Masson, Paris, 1959. p. 116.

21 I. M. Kolthoff et J. J. Lingane, Polarography, Vol. I, Interscience, New York, 1952, p. 202.

22 L. Meites, Polarographic Techniques, Interscience, New York, 1965, p. 228-229.

23 S. G. Mairanovskii dans P. Zuman (Ed.). Catalytic and Kinetic Waves in Polarography, Plenum Press, New York, 1968, p. 216.

24 I. M. Kolthoff et J. J. Lingane, Polarography, Vol. I, Interscience, New York, 2e edn., 1952, pp. $90-93$.

25 P. Zuman, dans P. Zuman et I. M. Kolthoff, Progress in Polarography, Vol. II, Interscience, New York, 1962, pp. 583-600.

26 L. Meites, Polarographic Techniques, Interscience; New York, 2e edn., 1965, pp. 138-140.

27 S. G. Mairanovskii dans P. Zuman (Ed.), Catalytic and Kinetic Waves in Polarography. Plenum Press, New York, 1968, p. 12 et pp. 85-93.

28 C. P. Andrieux, L. Nadjo et J. M. Savéant. J. Electroanal. Chem., 26 (1970) 147.

29 S. G. Mairanovskii, Dokl. Akad. Nauk SSSR, 110 (1956) 593.

30 J. M. Savéant et E. Vianello, C.R., 256 (1963) 2597. 
31 W. Kemula, Z. Grabowski et M. Kalinowski, Naturwiss., 47 (1960) 514.

32 S. G. Mairanovskii et V. N. Pavlov, Zh. Fiz. Khim., 38 (1964) 1804.

33 J. Volke, M. Naarova et V. Volkova, 21st C.I.T.C.E. Meeting, Prague, 1970.

34 C. P. Andrieux et J. M. Savéant, J. Electroanal. Chem., 26 (1970) 223.

35 S. G. Mairanovskii dans P. Zuman (Ed.), Catalytic and Kinetic Waves in Polarography, Plenum Press, New York, 1968, pp. 227-239.

36 S. G. Mairanovskii dans P. Zuman (Ed.), Catalytic and Kinetic Waves in Polarography, Plenum Press, New York, 1968, pp. 75-85.

37 R. Brdička et E. Knobloch, Z. Elektrochem., 47 (1941) 721.

38 R. Brdička, Z. Elektrochem., 48 (1942) 278.

39 R. Brdička, Collect. Czech. Chem. Commun., 12 (1947) 522. 